\section{Using genetics to develop new treatment possibilities for ovarian cancer}

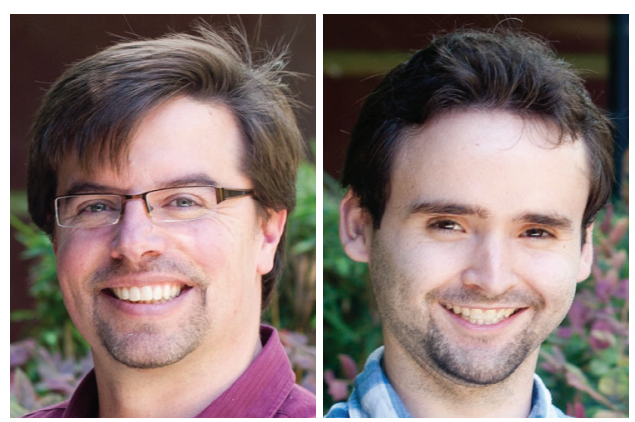

Joe R Delaney ${ }^{*, 1} \&$ Dwayne G Stupack ${ }^{1}$

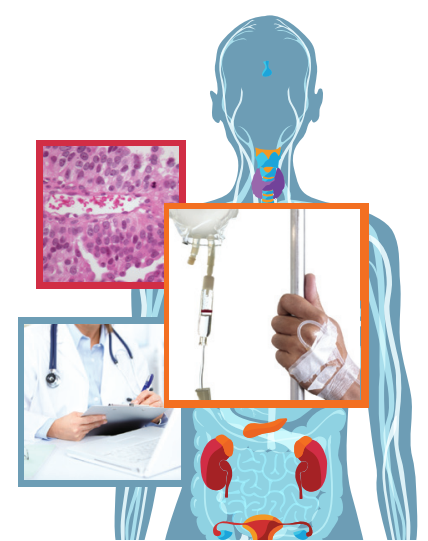

International Journal of Endocrine Oncology

\begin{abstract}
"As more genetic data become available, the first step toward therapy will be to fully integrate these data into an understanding of the biological network alterations within each tumor."
\end{abstract}

First draft submitted: 26 March 2017; Accepted for publication: 29 March 2017; Published online: 8 May 2017

Over the last 30 years or so, most of the advances in the treatment of ovarian cancer have come about largely as reformulations, or altered dosing, of classic chemotherapeutic agents such as cisplatin and taxol. Among patients with the most prevalent form of the disease, high-grade serous ovarian cancer (SOC), this has led to 5-year survival rates of $17-39 \%$ (stage III/IV) (SEER 2004-2010 data). Clearly, improvements are needed. Here, we briefly discuss major genomics findings in the context of improving treatment and describe what directions should be pursued in the near future to best enable the saving of lives.

In 2011, The Cancer Genome Atlas project released full exome sequencing data on 316 SOC tumors, somatic copynumber alteration (SCNA) data on 489 tumors, and mRNA transcriptome data on 489 tumors [1]. It was a huge undertaking, notable as the most comprehensive study in any tumor undertaken to that date - and well before human genomes had reached the US $\$ 1000$ per genome cost. Yet, the results were not unexpected relative to our prior understanding of this disease. Almost all tumors were inactivated in the TP53 gene. Somatic BRCA1 and BRCA2 mutations were present in 3.5 and $3.2 \%$ of tumors, respectively, and germline mutations were present in 9 and $8 \%$ of patients. A panel of other significant tumor suppressor or oncogene mutations, including NF1, RB1, CDK12, CSMD3, GABRA 6 and $F A T 3$, were found in $2-6 \%$ of patients. The results did more to support the idea that these genes could support tumorigenesis across many tissues than to provide insight into the biology of SOC; p53 mutations have been known for decades, as have BRCA mutations. However, the authors stated there was a 'remarkable degree of genomic disarray' in reference to the roughly two-thirds of an average SOC patient's genome impacted by SCNAs. The normal ' $2 \mathrm{~N}$ ' copy-number thus represented the minority of genes in SOC.

\section{KEYWORDS}

- genomics • ovarian cancer

- pathways 
"Many new therapies begin with basic science, and the genomic studies undertaken in the last few years have produced a radical shift in models used for studying serous ovarian cancer in the lab.”
Molecular tumor boards now review specific mutations with targeted therapeutics available, though mutation-targeted therapies remain rather limited in targets [2]. How have the genomic findings influenced first-line therapy for SOC in the 6 years since publication? The answer, to date, is not much. The first agents employed remain carboplatin and docetaxel. Therapies for recurrence range across other platinum/taxane combinations or doxorubicin derivatives. PARP inhibitors were already in development to target BRCA-mutant cells. These have found success with 15.5-month extension in progression-free survival in BRCA1/2 mutant tumors [3] and 9.1 months for tumors which were assayed to have homologous repair deficiency. This is predicted by synthetic lethality with $B R C A 1 / 2$ defective genotypes. Nonetheless, even tumors without the $B R C A$ mutation or homozygous deletion respond, with a progression-free survival extension of 5.4 months. Even with these exciting new results with hundreds of patients, clinicians caution that overall survival studies must follow this over a long period of time, given that PARP inhibitor resistance may also incur resistance to other secondline therapies [4]. A follow-up of the Phase II trial for Olaparib maintenance therapy indeed showed compaction of differences in overall survival: 34.9 months for BRCA-mutant tumors treated with olaparib compared with 30.2 months for placebo treated [5]. One may argue that the paucity of therapies emerging from these genetic studies implies limited value. Are they really worth the resources? We would argue 'yes' absolutely (and unequivocally). Any new therapies which may impact SOC will require a deep, multi-level understanding of this disease to enable integrative analysis, and the inevitable combination drug therapies necessary to defeat the disease.

A subsequent major sequencing study from the Australian Ovarian Cancer group investigated the genomic changes which occur following primary chemotherapy, in addition to more detailed analysis of structural variation [6]. They found half of SOC tumors were impaired in their homologous recombination DNA repair capacity via methylation of $B R C A 1$ promoters, or via $B R C A 1 / 2$ mutation in the germline or somatic tumor. Consistent with the TCGA findings, 12 tumors showed homologous repair deficiency by mRNA signature despite no known suppression of $B R C A 1 / 2$, potentially through Fanconi-anemia genes involved in homologous repair. Among recurrent chemotherapy-resistant tumor cells isolated from ascites, $B R C A 1 / 2$ reversions were observed, suggesting re-activation of homologous repair can endow SOC cells with a measure of chemoresistance, independent of changes in other key multidrug efflux transporters such as ABCB1. Despite the already high levels of SCNAs present in primary ovarian tumors [7], recurrent patients displayed an average increase in structural variants by nearly $60 \%$. Studies such as these enable an understanding of how SOC evolves before, during and after treatment. Ideal therapies should prevent the evolution of these resistance pathways or eliminate those cells likely to evolve one type of resistance with a complementary agent.

Many new therapies begin with basic science, and the genomic studies undertaken in the last few years have produced a radical shift in models used for studying SOC in the lab. Previously, the most widely used cell lines were A2780, IGROV1 and SKOV3 [8]. It turns out that none of these cell lines' genetics map closely to genuine SOC; hypermutated IGROV1 actually clusters most closely with melanoma by proteomics [9]. As such, new studies are focusing on model cell lines with closer to genuine genetics, such as OVKATE, CAOV4 or OVCAR3, a high SCNA, p53 mutated cell line with few of the other rare genetic driver mutations. Additionally, new media such as the OCMI media have been shown to allow for proliferation of cells which match far more closely to human disease, enabling the expansion of models to occur [10]. Having these appropriate models tested in cell death or microenvironment growth screens will better enable determination of targets for future pharmaceutical development. More targets and additional drugs must be added to the repertoire of clinically available gene-targeted therapeutics in order to achieve chemotherapy which overcomes tumor evolution.

Last, tumor heterogeneity is a factor which must not be ignored. As one of the most genetically heterogenous cancer types, despite its 'low' mutation rates [11], heterogeneity in SOC may be the main reason so few successful therapies exist [12]. A contributing factor is that the SCNAs which exist in these primary tumors already cover two-thirds of the genome and persist in the context of failed DNA repair pathways and cell cycle control. The very factors which give rise to the pervasive SCNAs also enable a tremendous diversity of SCNAs between cells within the same tumor and between metastatic tumors. In autopsy biopsy tumors, 11 independent reversions occurred across 18 metastatic sites [6]. 
Heterogeneity exists in premetastatic forms of the disease; serous tubal intraepithelial carcinomas already contain the majority of the SCNAs eventually found in late-stage tumors [13].

An excellent genomic study to track such diversity was completed in 2015 by the Brenton group, wherein they used whole-genome sequencing to capture minor alleles in tumor subpopulations and phylogenetic techniques to ascertain how SOC tumors evolve before and after therapy [11]. They found that after treatment, exceptionally infrequent clones overtake the tumor population after treatment. This supports a model of currently whack-a-mole treatment for SOC: as each new population arises, treatments only work if they work against that original subclone. One way to defeat such tumor evolution may be to treat potential subclones in anticipation of their expansion [14]. This may include attempting multiple targeted drugs during maintenance periods. It may also include earlier diagnostic methods such as circulating tumor cell diagnostics or cell-free circulating tumor DNA in routine blood tests, provided that the necessary $>99.9 \%$ specificity can be achieved to avoid false-positive diagnoses [15]. Either method would allow for limiting the heterogeneity of the cancer and increasing the likelihood that all tumor cells within a patient perish from therapy.

In our own experience, the best preclinical success has come about using heterogeneous treatment along with heterogeneous models with genetics close to real tumor genomic signatures. We developed a new algorithm to better sort through the enormous variation in SCNAs found in SOC [16]. The 'HAPTRIG' algorithm determined significantly disrupted pathways by surveying all heterozygous deletions and amplifications across 579 SOC tumors, scoring those changes based on haploinsufficiency data from model organisms, prioritizing genes which interact with many other genes (gene 'hubs'), and comparing hundreds of pathways for changes. The most significantly suppressed pathway found in SOC was autophagy, a cellular homeostasis pathway which enables a recycling of cellular detritus - big and small. Typically, such a pathway might be expected to be amplified in tumors. Its pervasive suppression suggested it may have deep roots in ovarian cancer development. Tumorigenicity is indeed increased upon autophagy suppression in animal models [17].

Yet the finding that autophagy was a grossly impacted pathway may also prove to be somewhat serendipitous. Available autophagy therapeutics target different points of the pathway, enabling the development of a 'heterogeneous treatment' which can have deep effect regardless of the individual pathway genes impacted. This prevents tumor escape, and would address the issue of SOC cell survival in minute populations containing resistant genetics. With the advent of a new SCNA-pathway target and a safety-oriented drug cocktail [18], we found exceptional suppression of tumor growth across mouse models. We are exploring options to translate these results into safety trials in patients, and efficacy in the clinic. Certainly we expect others around the world could similarly address this, particularly as the agents used are not expensive. As more genetic data become available, the first step toward therapy will be to fully integrate these data into an understanding of the biological network alterations within each tumor.

\section{Financial \& competing interests disclosure}

This work was funded in part by the NIH, grant number CA207729. The authors have no other relevant affliations or financial involvement with any organization or entity with a financial interest in or financial conflict with the subject matter or materials discussed in the manuscript apart from those disclosed.

No writing assistance was utilized in the production of this manuscript.

\section{References}

1 Cancer Genome Atlas Research N. Integrated genomic analyses of ovarian carcinoma. Nature 474(7353), 609-615 (2011).

2 Hughes PE, Caenepeel S, Wu LC. Targeted therapy and checkpoint immunotherapy combinations for the treatment of cancer. Trends Immunol. 37(7), 462-476 (2016).

3 Mirza MR, Monk BJ, Herrstedt J et al. Niraparib maintenance therapy in platinum-sensitive, recurrent ovarian cancer. N. Engl. J. Med. 375(22), 2154-2164 (2016).

4 Schram AM, Aghajanian CA, Hyman DM. Niraparib in recurrent ovarian cancer. $N$. Engl. J. Med. 376(8), 801 (2017).

5 Ledermann JA, Harter P, Gourley C et al. Overall survival in patients with platinumsensitive recurrent serous ovarian cancer receiving olaparib maintenance monotherapy: an updated analysis from a randomised, placebo-controlled, double-blind, Phase II trial. Lancet Oncol. 17(11), 1579-1589 (2016).

6 Patch AM, Christie EL, Etemadmoghadam $\mathrm{D}$ et al. Whole-genome characterization of chemoresistant ovarian cancer. Nature 521(7553), 489-494 (2015).

7 Zack TI, Schumacher SE, Carter SL et al. Pan-cancer patterns of somatic copy number alteration. Nat. Genet. 45(10), 1134-1140 (2013). 


\section{EDITORIAL Delaney \& Stupack}

8 Domcke S, Sinha R, Levine DA, Sander C, Schultz N. Evaluating cell lines as tumor models by comparison of genomic profiles. Nat. Commun. 4, 2126 (2013).

9 Gholami AM, Hahne H, Wu Z et al. Global proteome analysis of the NCI-60 cell line panel. Cell Rep. 4(3), 609-620 (2013).

10 Ince TA, Sousa AD, Jones MA et al. Characterization of twenty-five ovarian tumour cell lines that phenocopy primary tumours. Nat. Commun. 6, 7419 (2015).

11 Schwarz RF, Ng CK, Cooke SL et al. Spatial and temporal heterogeneity in high-grade serous ovarian cancer: a phylogenetic analysis. PLOS Med. 12(2), e1001789 (2015).
12 Burrell RA, Mcgranahan N, Bartek J, Swanton C. The causes and consequences of genetic heterogeneity in cancer evolution. Nature 501(7467), 338-345 (2013).

13 Eckert MA, Pan S, Hernandez KM et al. Genomics of ovarian cancer progression reveals diverse metastatic trajectories including intraepithelial metastasis to the fallopian tube. Cancer Discov. 6(12), 1342-1351 (2016).

14 Bozic I, Reiter JG, Allen B et al. Evolutionary dynamics of cancer in response to targeted combination therapy. Elife 2, e00747 (2013).

15 Bast RC, Jr., Brewer M, Zou C et al. Prevention and early detection of ovarian cancer: mission impossible? Recent Results Cancer Res. 174, 91-100 (2007).

16 Delaney JR, Patel CB, Willis KM et al. Haploinsufficiency networks identify targetable patterns of allelic deficiency in low mutation ovarian cancer. Nat. Commun. 8 , 14423 (2017).

$17 \mathrm{Qu}$ X, Yu J, Bhagat G et al. Promotion of tumorigenesis by heterozygous disruption of the beclin 1 autophagy gene. J. Clin. Invest. 112(12), 1809-1820 (2003).

18 Delaney JR, Patel C, Mccabe KE et al. A strategy to combine pathway-targeted low toxicity drugs in ovarian cancer. Oncotarget 6(31), 31104-31118 (2015). 